\title{
Communications
}

\section{Electromagnetic Performance Analysis of a Novel Monolithic Radome for Airborne Applications}

Raveendranath U. Nair and Rakesh M. Jha

\begin{abstract}
A monolithic half-wave hybrid variable thickness radome (hy$V T R$ ) is proposed here for airborne applications. The 3-D ray-tracing (in conjunction with aperture integration method), which is adapted here to evaluate the radome performance parameters, facilitates efficient analysis of antenna-radome interactions. The optimized power reflection $h y-V T R$ design is established to be superior to the conventional constant thickness radome (CTR) designs in terms of the EM performance.
\end{abstract}

Index Terms-Airborne radomes, monolithic half-wave, radome performance parameters, variable thickness radome (VTR) design.

\section{INTRODUCTION}

Modern airborne radomes, besides meeting the aerodynamic and payload requirements, have to also meet stringent electromagnetic (EM) performance specifications. This has often necessitated adopting novel and innovative radome design concepts. In a previous work reported elsewhere, these authors have proposed a hybrid variable thickness radome (hy-VTR) as a feasible concept to enhance the EM performance envelope considerably [1]; however this design too had the disadvantage of uncontrolled internal reflections, thereby leading to sidelobe level degradations and the image lobes. In order to elude such antenna pattern degradations, the authors proposed a novel A-sandwich radome design based on optimum power reflection [2]. The major disadvantage of such A-sandwich design is that the cross-polarization level is high particularly in the nose-cone sector, which limits its applications.

Nose-cone radomes for aircraft flying at supersonic and high mach speeds have to cater for extreme aerodynamic stresses. For such applications, monolithic half-wave wall configuration is generally preferred to other radome wall structures. The study presented in [2] is further extended to a novel monolithic half-wave hy-VTR design, based on the optimization of power reflection. Hence the spurious internal reflections within the radome are minimized, which offers minimal degradations to antenna pattern characteristics. Radome performance parameters are evaluated for the proposed design based on aperture integration method in conjunction with ray-tracing. It will be shown through EM analysis that important performance parameters such as the power transmission, cross-polarization and boresight error (BSE) are also significantly improved.

\section{EM DESIGN CONSIDERATIONS FOR THE RADOME}

\section{A. Geometry of the Antenna-Radome System}

The geometry of the hybrid shaped radome is shown in Fig. 1. A hybrid cone-ogive radome considered here is of typical dimensions

Manuscript received May 30, 2008; revised May 21, 2009. First published June 30, 2009; current version published November 04, 2009.

The authors are with the Computational Electromagnetics Lab, Aerospace Electronics and Systems Division, National Aerospace Laboratories (NAL-CSIR), Council of Scientific and Industrial Research, Bangalore 560 017, India (e-mail: raviunair@nal.res.in; jha@ nal.res.in).

Digital Object Identifier 10.1109/TAP.2009.2026595

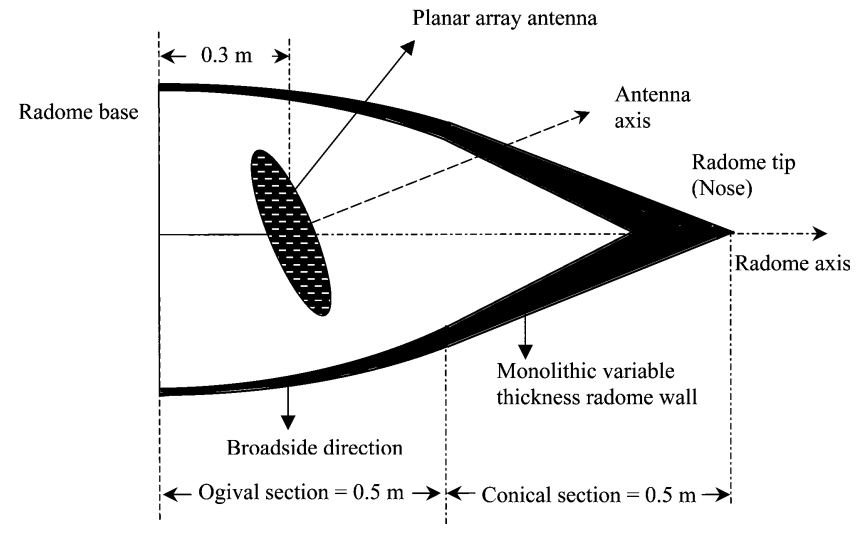

Fig. 1. Monolithic half-wave hybrid radome.

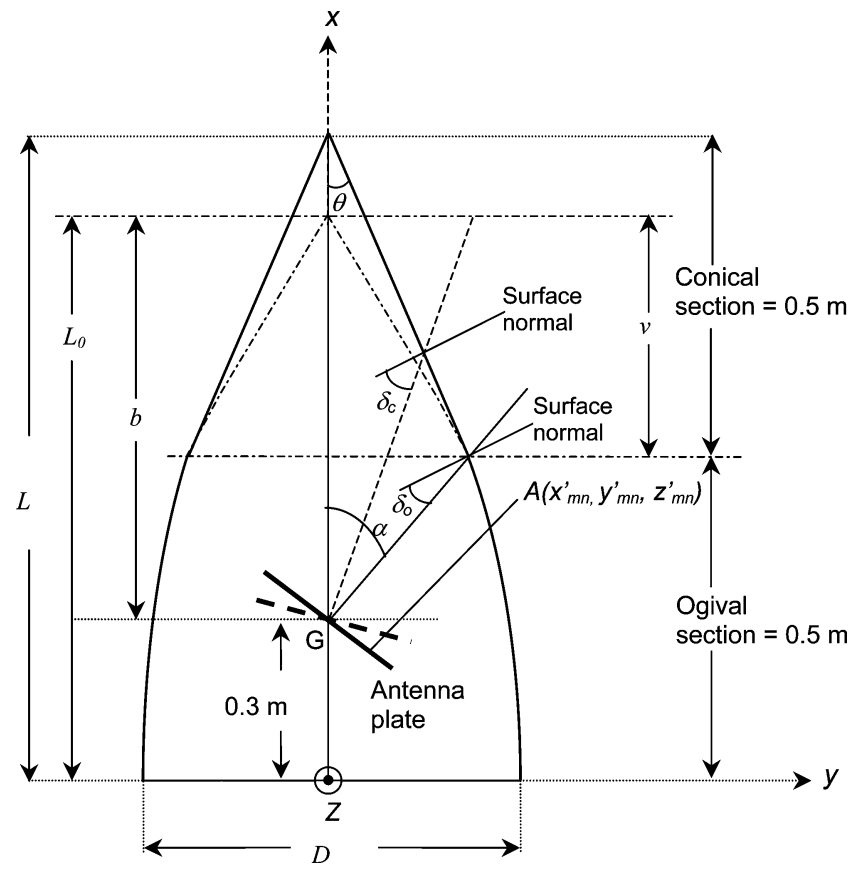

Fig. 2. Schematic for the determination of angle of incidence.

(height $=1 \mathrm{~m}$; base diameter $=0.5 \mathrm{~m}$ ) for airborne applications. The front conical portion of the radome (height $=0.5 \mathrm{~m}$ ), is followed by a tangent-ogival section of identical height. The radome houses a monopulse planar array antenna (diameter $=0.2 \mathrm{~m}$ ), which is assumed to be located at $0.3 \mathrm{~m}$ from the base of the radome. The polarization of the antenna is vertical and its operating centre frequency is $9.4 \mathrm{GHz}$ with a frequency bandwidth of $800 \mathrm{MHz}$. The radiating elements of the array antenna are rectangular slots spaced nearly halfwavelength apart. The antenna is fixed to a gimbal system, which facilitates the scans in azimuth and elevation planes without rotational offsets during radar operation. The reference Cartesian coordinate system $(x, y, z)$ is at the center of the radome base (Fig. 2).

The gimbal system is located at a distance $d_{g}$ from the radome base, which is the realistic case in most of the airborne radar systems (Fig. 2). 
TABLE I

ThickNess PRofile For Monolithic Half-WaVe Radome Wall

\begin{tabular}{|c|c|}
\hline Antenna Scan Angle & $\begin{array}{c}\text { Wall Thickness* } \\
\text { (in mm) }\end{array}$ \\
\hline $0^{\circ}$ & 9.12 \\
\hline $10^{\circ}$ & 8.92 \\
\hline $20^{\circ}$ & 8.7 \\
\hline $30^{\circ}$ & 8.4 \\
\hline $40^{\circ}$ & 8.2 \\
\hline $50^{\circ}$ & 8.0 \\
\hline $60^{\circ}$ & 8.0 \\
\hline $70^{\circ}$ & 8.0 \\
\hline $80^{\circ}$ & 8.0 \\
\hline $90^{\circ}$ & 8.0 \\
\hline
\end{tabular}

* The wall thickness at all angles except at the critical nosecone sector $\left(0^{\circ}-10^{\circ}\right)$ is truncated at the first decimal place due to typical practical fabrication considerations. This has resulted in identical values in the range $\left(50^{\circ}-90^{\circ}\right)$.

Here $G\left(d_{g}, 0,0\right)$ represents the centre of the gimbal mechanism. The coordinate system at the centre of the antenna plate is represented by $\left(x^{\prime}, y^{\prime}, z^{\prime}\right)$. The location of the $m n^{\text {th }}$ radiating slot on the planar array antenna is represented by $A\left(x_{m n}^{\prime}, y_{m n}^{\prime}, z_{m n}^{\prime}\right)$.

\section{B. EM Design Aspects of Radome Wall}

The thickness profile of radome wall (glass composite: relative permittivity, $\varepsilon_{r}=4.0$; electric loss tangent, $\left.\tan \delta_{\mathrm{e}}=0.015\right)$ is generated based on the determination of angle of incidence. The outer surface of the radome is coated with a typical radome paint $\left(\varepsilon_{r}=3.46\right.$; electric loss tangent, $\tan \delta_{e}=0.068$ ) of thickness $0.2 \mathrm{~mm}$. The schematic diagram for the determination of angle of incidence is shown in Fig. 2. Let $L$ and $D$ be the length and diameter of the base of the hybrid shaped radome respectively. Here the angle of incidence is the angle between the antenna axis and the surface normal drawn at the point of intersection of antenna axis with the radome wall.

The conical section of the radome has a semi-apex angle $\theta$. Let $\alpha$ be the antenna scan angle measured w.r.t. z-axis. Then the angle of incidence corresponding to the conical section is given by

$$
\delta_{c}=90-(\theta+\alpha)
$$

Let $L_{0}$ be the notional height (Fig. 2) and $R$ be the radius of curvature of the tangent-ogive section of the radome respectively. For the determination of incidence angles, the ogival section is extended up to its tip (represented by dashed line). Let " $v$ " be the vertical distance of the ogival tip from the intercept point of antenna axis with the ogival portion of the radome wall. The antenna plate is located at a distance ' $b$ ', from the tip of the ogive.

Here

$$
R=\frac{4 L_{0}^{2}+D^{2}}{4 D}
$$

The antenna scan angle is given by

$$
\alpha=90-\tan ^{-1}\left(\frac{b-v}{\sqrt{R^{2}-\left(L_{0}-v\right)^{2}}-B}\right)
$$

where $B=R-(D / 2)$.
Equation (3) gives the values of " $v$ " corresponding to $\alpha=$ $0^{\circ}, 5^{\circ}, 10^{\circ}, \ldots, 90^{\circ}$. Substituting the value of " $v$ " in the following expression gives the angle of incidence corresponding to antenna scan angles for the tangent-ogive section:

$\delta_{o}=\tan ^{-1}\left(\frac{b-v}{\sqrt{R^{2}-\left(L_{0}-v\right)^{2}}-B}\right)-\sin ^{-1}\left(\frac{L_{0}-v}{R}\right)$.

The wall thickness profile is generated at the centre frequency $9.4 \mathrm{GHz}$ for optimum reflection based on the equivalent transmission line method described in [3]. It optimizes the power reflection parameter, such that the VSWR as well as the sidelobe level degradation of the antenna-radome system are minimized. This results in a wall thickness design profile (Table I) as a function of antenna scan angle, which varies from $8.0 \mathrm{~mm}$ (radome base) to $9.12 \mathrm{~mm}$ (radome tip).

\section{THE ANTENNA-RADOME INTERACTION}

The interaction of the slotted waveguide planar array antenna with the hy-VTR configuration is carried out by a 3-D ray-tracing procedure in conjunction with aperture integration (AI) method [4]. The radome performance parameters are evaluated theoretically by considering the antenna-radome system w.r.t. the antenna alone reference. The EM analysis procedure includes: (i) fixing of radome coordinates; (ii) determination of the space phase terms, along with its transformation to the radome coordinates; and (iii) obtaining EM performance parameters of the radome (e.g., Co-pol power transmission, X-pol, BSE etc.) as a function of antenna scan angle. The antenna is assumed to be in the receiving mode for the purpose of computation of radome performance parameters. The initial step involves the transformation of aperture points on the antenna surface to the coordinates of the radome after incorporating the gimbal rotations (Fig. 2). The gimbal system selected indicates the position of a target in terms of scan angles in azimuth and elevation planes. Let $A z$ and $E l$ represent the scan angles in the azimuth (xz) and elevation (xy) planes respectively. The preliminary step in this analysis is the computation of the transformation due to the angular rotations corresponding to gimbal orientations [4]. For the gimbal system considered here, there are no rotational offsets. Hence the transformation of the $m n^{\text {th }}$ radiating slot on the planar array antenna to the coordinates of radome $(x, y, z)$ is given by (5), shown at the bottom of the following page. The gimbal system is located at a distance $d_{g}$ 


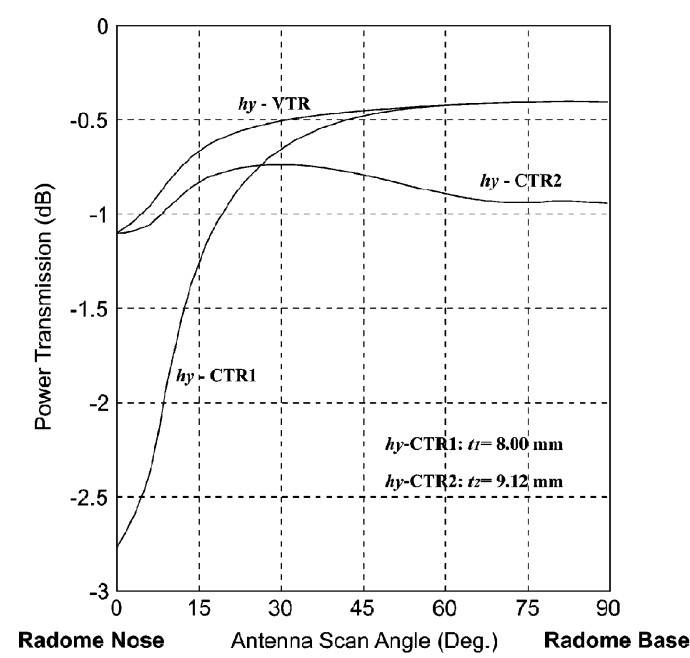

(a)

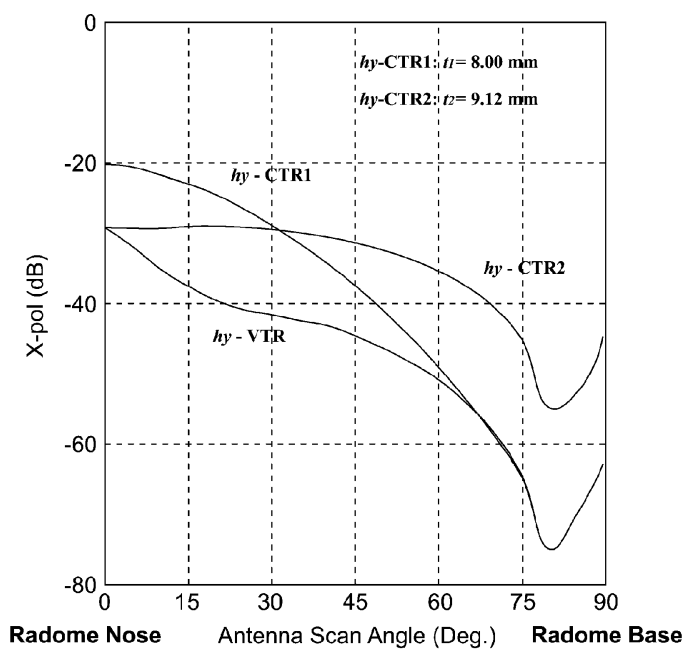

(b)

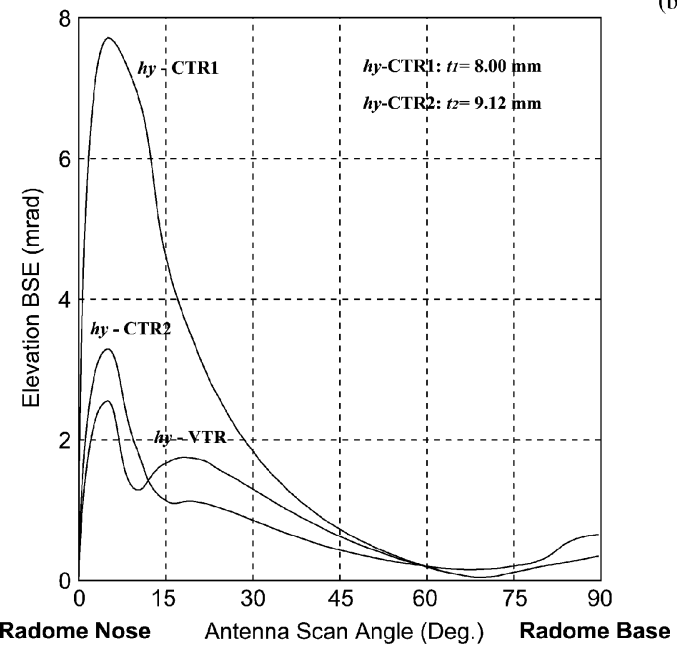

(c)

Fig. 3. (a) Co-pol power transmission versus antenna scan angle for hy-VTR and hy-CTR designs. (b) X-pol power transmission versus antenna scan angle for hy-VTR and hy-CTR designs. (c) Elevation BSE versus antenna scan angle for hy-VTR and hy-CTR designs.

from the centre of the radome base. Then transforming the results linearly to the coordinates of the radome gives (6), shown at the bottom of the page. Thus the location of each rectangular slot of the planar array is transformed into the radome coordinates. The ray vectors from the finite rectangular slots are transformed in the look direction (parallel to the antenna axis). The corresponding angles of incidence are deter- mined based on 3-D ray-tracing by projecting rays on to the radome surface and determining the ray intercept points. The space phase terms of the antenna far-field are then computed from the hy-VTR contour profile based on the selected coordinate system.

For each ray intercepting the wall, corresponding to each radiating slot of the antenna, both the co-pol and cross-pol voltage transmission

$$
\left[\begin{array}{l}
x_{m n} \\
y_{m n} \\
z_{m n}
\end{array}\right]=\left[\begin{array}{ccc}
\cos A z \cos E l & -\cos A z \sin E l & -\sin A z \\
\sin E l & \cos E l & 0 \\
\sin A z \cos E l & -\sin A z \sin E l & \cos A z
\end{array}\right]\left[\begin{array}{l}
x_{m n}^{\prime} \\
y_{m n}^{\prime} \\
z_{m n}^{\prime}
\end{array}\right] .
$$

$$
\left[\begin{array}{l}
x_{m n} \\
y_{m n} \\
z_{m n}
\end{array}\right]=\left[\begin{array}{ccc}
\cos A z \cos E l & -\cos A z \sin E l & -\sin A z \\
\sin E l & \cos E l & 0 \\
\sin A z \cos E l & -\sin A z \sin E l & \cos A z
\end{array}\right]\left[\begin{array}{c}
x_{m n}^{\prime} \\
y_{m n}^{\prime} \\
z_{m n}^{\prime}
\end{array}\right]+\left[\begin{array}{c}
d_{g} \\
0 \\
0
\end{array}\right] .
$$




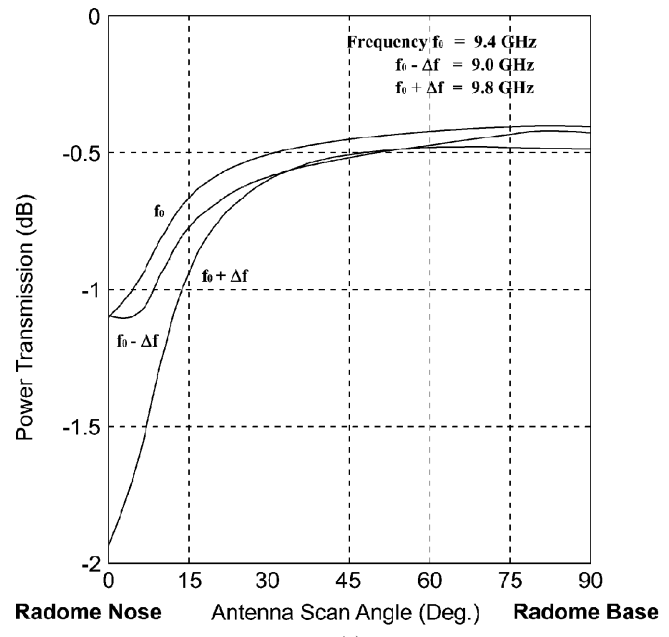

(a)

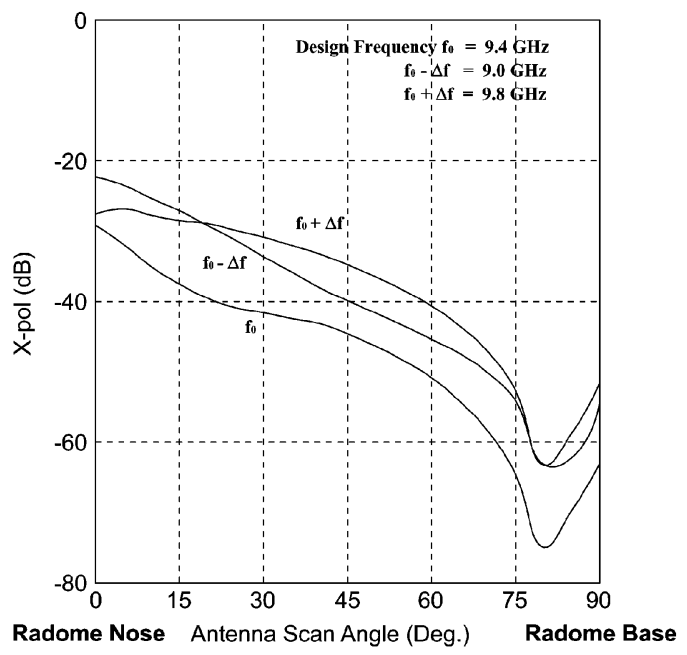

(b)

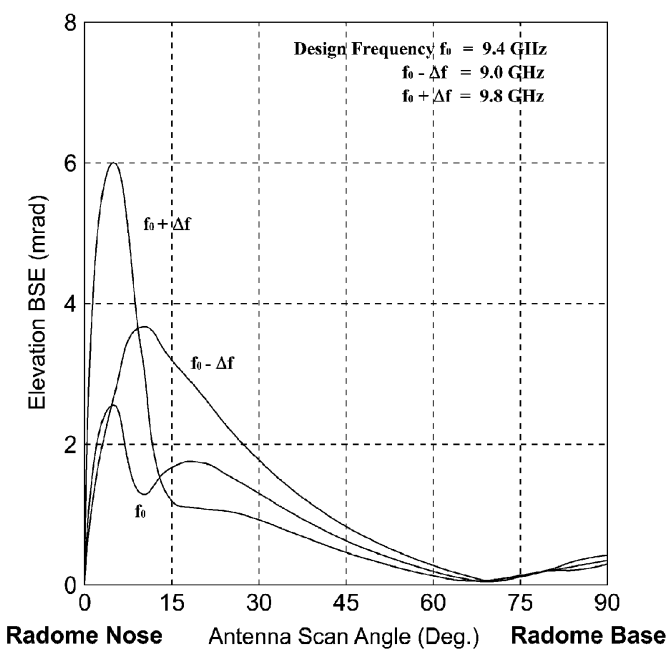

(c)

Fig. 4. (a) Power transmission characteristics of hy-VTR over the frequency range. (b) Cross-pol power transmission characteristics of hy-VTR over the frequency range. (c) Elevation boresight error characteristics of hy-VTR over the frequency range.

coefficients are computed. This involves the angle of incidence, radome wall thickness, dielectric parameters of the radome wall, wavelength and polarization. The antenna aperture integration is performed to determine the receiving port voltage from the pattern look direction for the antenna-radome system. The normalized array pattern for the antenna-radome system is given by [4]

$$
E_{A R}=\frac{\sum_{m=1}^{M} \sum_{n=1}^{N} F_{m n}^{a} T_{m n} e^{-j\left(k_{x} x+k_{y} y+k_{z} z\right)}}{\sum_{m=1}^{M} \sum_{n=1}^{N} F_{m n}^{a}}
$$

where $T_{m n}$ and $F_{m n}^{a}$ are the transmission coefficient and aperture distribution for the $m n^{\text {th }}$ radiating slot respectively. The aperture distribution characteristics of the rectangular slots of the antenna are incorporated in the computation of co-pol and cross-pol power transmission parameters for the antenna-radome system.

For the monopulse antenna, let $\Delta V_{A z}$ and $\Delta V_{E l}$ be the receiving port voltages corresponding to azimuth and elevation difference channels, respectively. $\Delta V_{A z}$ and $\Delta V_{E l}$ for the given pattern look direction are obtained by using $F_{m n}^{a}=\boldsymbol{S i n}\left(\pi x_{m n} / r_{\mathrm{a}}\right)$ and $F_{m n}^{a}=\boldsymbol{S i n}\left(\pi y_{m n} / r_{\mathrm{a}}\right)$ respectively in the (7). Here $r_{\mathrm{a}}$ represents the radius of the antenna. Then the boresight errors for azimuth and elevation cases are given by [5]

$$
\begin{aligned}
B S E_{A z} & =\frac{\Delta V_{A z}}{K_{A z}} \\
B S E_{E l} & =\frac{\Delta V_{E l}}{K_{E l}}
\end{aligned}
$$

where $K$ represents the monopulse antenna sensitivity that depends on the port voltages and azimuth/elevation offsets. The same procedure is adopted for the EM performance predictions of two extreme hy-CTR designs. Since the hy-VTR thickness profile varies from $8.00 \mathrm{~mm}$ to $9.12 \mathrm{~mm}$, two extreme hy-CTR designs [hy-CTR1: $t_{1}=8.00 \mathrm{~mm}$ (constant), and hy-CTR2: $t_{2}=9.12 \mathrm{~mm}$ (constant)] are considered.

\section{NUMERICAL RESULTS AND DISCUSSION}

The hy-VTR design proposed here is compared with the hy-CTR designs, since they are apparently simple to fabricate. As evident from the performance curves for various EM parameters [Fig. 3(a)-(c)], the hy-VTR design offers minimal amplitude and phase distortions.

The co-pol power transmission for the hy-VTR and hy-CTR designs are shown in Fig. 3(a). For hy-CTR1 design, this parameter is much lower in the critical nose-cone sector and is clearly not acceptable Similarly, for the hy-CTR2 design, the power transmission efficiency 
degrades at larger antenna scan angles. Fig. 3(a) clearly indicates that power transmission efficiency for the hy-VTR design is superior to that of both the extreme hy-CTR design cases.

The depolarization characteristics of the hy-VTR and hy-CTR designs are shown in Fig. 3(b). In the case of hy-VTR design, the X-pol power transmission is well below $-30 \mathrm{~dB}$ throughout the antenna scan range and it is superior to the hy-CTR designs. For the hy-CTR1 design, X-pol power transmission is much higher, especially around the nose-cone region, which is undesirable for airborne radar systems. Furthermore, the hy-VTR design proposed here has far superior blending characteristics for X-pol than those reported in [1].

The elevation BSE characteristics are shown in Fig. 3(c). It is readily inferred that the BSE for the hy-VTR design is again, better than that of hy-CTR designs. The BSE for the hy-CTR1 design shows a sharp variation around the nose-cone sector of the radome, which is not desirable. Further, the hy-VTR design, though not optimized for the BSE, has resulted in acceptable BSE performance as well.

In order to analyze the frequency response of the hy-VTR, the EM performance parameters are computed at the end-frequencies of the given radar antenna [Fig. 4(a)-(c)]. It is observed that the deterioration of power transmission is more at $9.8 \mathrm{GHz}$ than that at $9.0 \mathrm{GHz}$ in the critical nose-cone sector [Fig. 4(a)]. Regarding the cross-pol transmission characteristics, it is high at $9.0 \mathrm{GHz}$ as compared to that at $9.8 \mathrm{GHz}$ in the nose-cone sector [Fig. 4(b)]. Even though the boresight error at $9.8 \mathrm{GHz}$ shows a sharp increase in the nose-cone sector as compared to that at $9.0 \mathrm{GHz}$ [Fig. 4(c)], it is well within the acceptable limits.

\section{CONCLUSION}

The EM performance parameters are evaluated for a novel hy-VTR design based on 3-D ray-tracing with aperture integration method. The hy-VTR design based on optimized power reflection offers superior EM characteristics due to the minimization of internal reflections. The EM analysis for the present work is more accurate than the conventional approach due to the incorporation of antenna and radome as a system, and the finite-dimensional nature of the antenna. A comparative study of radome performance parameters establishes the superior electromagnetic performance of the hy-VTR design over the conventional constant thickness designs.

\section{REFERENCES}

[1] R. U. Nair and R. M. Jha, "Novel design for a monolithic hybrid variable thickness radome (hy-VTR)," in Proc. IEEE Antennas Propag. Soc. Int. Symp., 2004, pp. 878-881.

[2] R. U. Nair and R. M. Jha, "Novel A-sandwich radome design for airborne applications," Elect. Lett., vol. 43, pp. 787-789, Jul. 2007.

[3] R. H. J. Cary, "Radomes," in The Handbook of Antenna Design. London, U.K.: Peter Peregrinus, 1983.

[4] D. J. Kozakoff, Analysis of Radome-Enclosed Antennas. Norwood, MA: Artech House, 1997.

[5] K. Siwiak, T. B. Dowling, and L. R. Lewis, "Boresight errors induced by missile radomes," IEEE Trans. Antennas Propag., vol. AP-27, pp. 832-841, Nov. 1979.

\section{Enhanced Low-Angle GPS Coverage Using Solid and Annular Microstrip Antennas on Folded and Drooped Ground Planes}

\author{
Hussain M. Al-Rizzo, Ken G. Clark, Jim M. Tranquilla, \\ Rami A. Adada, Taha A. Elwi, and Daniel Rucker
}

\begin{abstract}
Folded and drooped microstrip antennas are investigated in this communication for their potential applications in GPS marine navigation. Numerical and experimental results are reported to identify the effects of the percentage of the patch extending around to the folded side, position, and angle of the bend on the performance of the proposed antennas in comparison to the conventional flat counterparts. The folded antennas provide marginally improved 3-dB beam width and excellent phase center stability without degrading the bore-sight gain. A novel drooped square annular element operating in the $\mathrm{TM}_{30}$ mode is proposed and validated both numerically and experimentally. The drooped annular antenna is shown to have substantially improved above-horizon coverage to suit applications requiring acquisition of satellites from horizon to horizon with a pattern ripple less than $2 \mathrm{~dB}$ over the upper hemisphere and with an impedance bandwidth of $2 \%$. The polarization rejection is marginally degraded at bore-sight. At the horizon, the cross component becomes dominant by $1.5 \mathrm{~dB}$.
\end{abstract}

Index Terms-Drooped microstrip antenna, GPS, low-elevation pattern coverage, marine navigation, pyramidal ground plane, $\mathbf{T M}_{30}$ square annular patch.

\section{INTRODUCTION}

It is well known that a conventional microstrip antenna, typically mounted on a flat ground plane, suffers a remarkable decrease in gain toward low-elevation angles $\left(10^{\circ}\right.$ to $30^{\circ}$ above the horizon). Therefore, interest exists for improving this inherent coverage performance to allow for early signal acquisition from rising satellites, to avoid loss of contact and cycle slips, and to maintain signal continuity and proper system's dilution of precision. The research reported in this communication focuses on real-time dynamic GPS marine applications. The design goal is to compensate for the motion (pitch and roll) by extending coverage over a wide range of elevation angles including $20^{\circ}$ below the antenna's horizon plane [1].

Some work has been reported on improving the hemispherical coverage of a crossed dipole antenna using a cylindrical ground plane with a flat elevated center surrounded by sloping sides [2]-[4]. The structure was found to be successful in improving the radiation pattern at low-elevation angles. Additional elements were also examined such as monofilar and quadrifilar helices [5], although none achieved the same degree of pattern modification as the crossed dipoles. This is attributed

Manuscript received July 28, 2008; revised March 19, 2009. First published September 11, 2009; current version published November 04, 2009. This work was supported in part by the National Science Foundation (NSF) EPSCoR under Grant EPS-0701890.

H. M. Al-Rizzo is with the Systems Engineering Department, Donaghey College of Engineering and Information Technology, University of Arkansas at Little Rock, Little Rock, AR 72204-1099 USA (e-mail: hmalrizzo@ualr.edu).

R. A. Adada, T. A. Elwi, and D. Rucker are with the Applied Science Department, Donaghey College of Engineering and Information Technology, University of Arkansas at Little Rock, Little Rock, AR 72204-1099 USA (e-mail: hmalrizzo@ualr.edu).

K. G. Clark and J. M. Tranquilla are with EMR Microwave Technology Corporation, Fredericton NB E3X 1N2, Canada.

Color versions of one or more of the figures in this communication are available online at http://ieeexplore.ieee.org.

Digital Object Identifier 10.1109/TAP.2009.2032104 